\title{
Consideration for Lumbar Disc Degeneration and Herniation in Sports
}

Ziva Majcen

Rehabilitation Centre Spa Zrece, Zrece, majcen.ziva10@gmail.com

Tanja Rauter Pungartnik

University of Primorska, Science and Research Centre, Institute for Kinesiology Research, Koper

Nejc Sarabon

S2P Ltd., Laboratory for Motor Control and Motor Behaviour, Ljubljana

Follow this and additional works at: https://nsuworks.nova.edu/ijahsp

Part of the Medicine and Health Sciences Commons

\section{Recommended Citation}

Majcen Z, Pungartnik T, Sarabon N. Consideration for Lumbar Disc Degeneration and Herniation in Sports. The Internet Journal of Allied Health Sciences and Practice. 2014 Apr 01;12(2), Article 6.

This Manuscript is brought to you for free and open access by the College of Health Care Sciences at NSUWorks. It has been accepted for inclusion in Internet Journal of Allied Health Sciences and Practice by an authorized editor of NSUWorks. For more information, please contact nsuworks@nova.edu. 


\title{
Consideration for Lumbar Disc Degeneration and Herniation in Sports
}

\begin{abstract}
Lower-back related injuries in an athletic population are common and may have severe implications for the athletes sporting career. The focus of this work was to review epidemiology, intervention strategies, and recommendations for return to practice and competition for athletes with lumbar disc degeneration and herniated disc. Conservative treatments for discogenic degeneration and lumbar spine herniation are generally sufficient for athletic populations to restore functional movement. Athletes that fail to respond to conservative measures may consider lumbar discectomy, with high levels of return to precompetition levels observed following effective surgery. Surgical procedures such as spinal fusion and disc replacement require careful consideration, and should only be recommended if the athlete fails to respond to vigorous conservative treatments. Spinal disc implants have durability and mechanical loading concerns, whereas spinal fusion may ultimately prevent participation in contact sports. Specific guidelines for return to practice and competition remain poorly defined, thus future investigations should address this gap in knowledge. Return to sports should be carefully recommended after the athlete demonstrates sufficient improvements in neuro-physiological, biomechanical, and psychological domains. The correct rehabilitative exercise programme is vital to the rehabilitation of athletes following injury, and should consider frequent monitoring of pain, range of motion and muscle function.
\end{abstract}

\section{Author Bio(s)}

- Ziva Majcen, BSc, Rehabilitation Centre Spa Zrece, Zrece

- Tanja Rauter Pungartnik, MD, University of Primorska, Science and Research Centre, Institute for Kinesiology Research, Koper

- Nejc Sarabon, PhD, S2P Ltd., Laboratory for Motor Control and Motor Behaviour, Ljubljana 


\title{
IJAHSP

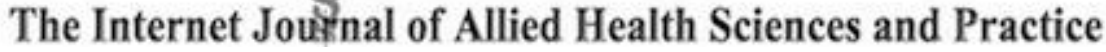 \\ Dedicated to allied health professional practice and education \\ http://ijahsp.nova.edu Vol. 12 No. 2 ISSN 1540-580X
}

\section{Consideration for Lumbar Disc Degeneration and Herniation in Sports}

\author{
Ziva Majcen, $\mathrm{BSc}^{1}$ \\ Tanja Rauter Pungartnik, MD2 \\ Nejc Sarabon, $\mathrm{PhD}^{3}$
}

1. Rehabilitation Centre Spa Zrece, Zrece

2. University of Primorska, Science and Research Centre, Institute for

Kinesiology Research, Koper

3. S2P Ltd., Laboratory for Motor Control and Motor Behaviour, Ljubljana

Slovenia

\begin{abstract}
Lower-back related injuries in an athletic population are common and may have severe implications for the athletes sporting career. The focus of this work was to review epidemiology, intervention strategies, and recommendations for return to practice and competition for athletes with lumbar disc degeneration and herniated disc. Conservative treatments for discogenic degeneration and lumbar spine herniation are generally sufficient for athletic populations to restore functional movement. Athletes that fail to respond to conservative measures may consider lumbar discectomy, with high levels of return to precompetition levels observed following effective surgery. Surgical procedures such as spinal fusion and disc replacement require careful consideration, and should only be recommended if the athlete fails to respond to vigorous conservative treatments. Spinal disc implants have durability and mechanical loading concerns, whereas spinal fusion may ultimately prevent participation in contact sports. Specific guidelines for return to practice and competition remain poorly defined, thus future investigations should address this gap in knowledge. Return to sports should be carefully recommended after the athlete demonstrates sufficient improvements in neuro-physiological, biomechanical, and psychological domains. The correct rehabilitative exercise programme is vital to the rehabilitation of athletes following injury, and should consider frequent monitoring of pain, range of motion and muscle function.
\end{abstract}

\section{GENERAL OVERVIEW OF DISC DEGENERATION \& HERNIATION}

In order to understand how intervertebral disc degeneration and herniation may occur, it is important to highlight key structures and functions related to the intervertebral connections/space, especially the discs. Intervertebral discs have an important biomechanical role within the spine, as they allow for motion between the spinal segments while dispersing compressive, sliding, and torsional forces. ${ }^{1}$

These discs are complex structures that consist of a thick outer ring of fibrous cartilage termed the annulus fibrosus, which surrounds a more gelatinous core known as the nucleus pulposus; the nucleus pulposus is contained within the cartilage end plates inferiorly and superiorly. The intervertebral disc is the primary load-bearing structure in the spinal motion segment and allows transmission and absorption of forces arising from body weight and muscle activity. ${ }^{2}$ In order to perform this function, it relies largely on the structural condition of the disc nucleus, annulus, and the vertebral endplate. Under normal conditions, and when the disc is functioning optimally, forces are dispersed across the disc evenly. However, structural deterioration of the disc can decrease the ability of the disc to withstand extrinsic forces, as forces are no longer transmitted proportionally.

Degeneration of the intervertebral disc is strongly associated with back pain. Disc degeneration involves structural disruption and cell-mediated changes in composition. Mechanical loading, traumatic, nutritional, and genetic factors all may play a role in disc degeneration. ${ }^{3}$ The lower lumbar region endures the heaviest mechanical forces, and thus failure of discs is more common compared to other regions. In comparison to other musculoskeletal tissues, discs have a tendency to degenerate earlier, with 
adolescents presenting signs between the ages of 11 to 16 years. ${ }^{4}$ With increasing age there is an associated degeneration of the intervertebral discs observed. ${ }^{5}$

In order to understand how mechanical loads may predispose an athlete to degenerative disc disease and herniation, the movements of the lumbar spine must be identified. Other factors in addition to mechanical loads contribute to disc degeneration, but these are not within the scope of this review. Essentially, the most pronounced movements of the lumbar spine are flexion and extension in the sagittal plane; other motions are axial rotation and lateral bending. ${ }^{6}$ However, while the disc may be at risk of injury in all fundamental planes of motion, it is particularly susceptible during repetitive flexion, or hyperflexion, combined with lateral bending or rotation. ${ }^{7}$

Chronic repetitions of these movements as well as traumatic events can lead to injury of the intervertebral disc, both of which are observed in sports. When these movements are combined with excessive axial compression, there is a concern for the integrity of the internal structure of the disc. Axial compression may derive from lumbar spine musculature, body weight, and external forces. Muscular forces developed during tasks such as lifting may also contribute to axial compression loads. Athletes are commonly exposed to high loading conditions.

An analysis of world-class power lifters calculated compressive loads between $18800 \mathrm{~N}$ and $36400 \mathrm{~N}$ acting at the L3-4 motion segment. ${ }^{8}$ Elite level football linesmen have also been shown to present time-related hypertrophy of the disc and changes in vertebrae endplate in response to the repetitive high loading and axial stress. ${ }^{9}$ More recently, long distance runners have been shown to undergo significant strain to the intervertebral disc, indicated by a reduction in disc height. ${ }^{10}$ When highly susceptible movements and loading are combined, they can cause loss of restraint of the nucleus by producing damage to the endplate or annulus areas of the disc. ${ }^{11}$

As mentioned earlier, the condition of the disc nucleus, annulus, and the vertebral endplate are important to the load bearing capacity of the intervertebral discs. The nucleus has an important role in dispersing repetitive loading forces from the disc by distributing forces transversally to the annulus, and in doing so, the nucleus decreases the risk of mechanical failure. ${ }^{1}$ Over time, these structural deficiencies in the annulus or endplate may lead to global disc degeneration, which could further progress to lumbar disc herniations. ${ }^{12}$

According to Modic and Ross, herniation refers to a localised displacement of the nucleus or fragmented annular tissue beyond the intervertebral disc space. ${ }^{3}$ During a herniation, the nucleus pulposus places pressure on weakened area of the annulus, and proceeds through the weakened sites on the annulus where it ultimately forms a herniation. ${ }^{13}$ In accordance to this, it appears that some form of disc degeneration exists before the disc can actually herniate. ${ }^{14}$

\section{EPIDEMIOLOGY}

It is estimated that $30 \%$ of athletic participants report lower back pain (LBP). ${ }^{15}$ Athletes are involved in complex movements during sporting events that may predispose the lumbar spine to greater mechanical loads than that of non-athletes. The greatest prevalence of LBP is observed with athletes that frequently perform hyperextension (extension of the spine backwards, beyond the anatomical position) manoeuvres and those engaged in repetitive flexion (forward bending), axial loading/jumping, and twisting. For example, athletes engaged in sports such as diving and gymnastics are required to commonly perform repeated hyperextension movements and are thus associated with higher incidence of spinal disorders. ${ }^{16}$ Athletes involved in sports with emphasis on prolonged and/or repetitive flexion such as skiing and cycling may also be at risk of lower back problems.

Furthermore, those who participate in longer and intense training regimes appear to be at higher risk of spinal pathologies, as do those involved in impact sports. Various radiographic techniques reveal that athletes present more cases of disc degeneration than the general population.

Sward and colleagues investigated disc degeneration in 24 male elite gymnasts and 16 non-athletes and found that gymnasts were at a higher risk of disc degeneration than non-athletes. ${ }^{17}$ Elite level female gymnasts have also been associated with increased degenerative discs. ${ }^{18}$ More recently, Ong et al observed that the prevalence of lumbar intervertebral disc degeneration was greater in Olympic athletes when compared to the normal population, although prevalence for disc degeneration in the nonathletes was obtained from published literature. ${ }^{19}$

Various degrees of disc displacements were found in 18 of the 31 Olympic subjects. Alyas et al found that elite level tennis players show high incidence of abnormalities in the lower lumbar spine region. ${ }^{20}$ Magnetic resonance imaging (MRI) revealed that 28 of 33 adolescent tennis players presented clinical symptoms, ranging from disc degeneration, disc herniation, pars

(c) The Internet Journal of Allied Health Sciences and Practice, 2014 
lesions, and facet joint arthropathy. Contrary to findings that suggest degenerative disc disease is more prevalent in athletes than non-athletes, Capel et al found that that female flamenco and ballet dancers had a lower prevalence of disc degeneration as measured by MRI when compared to age matched non-athletic controls. ${ }^{21}$ This suggests that the type of physical activity influences the prevalence of disc degeneration in an athletic population. This is supported by the historical cohort study, which found that sports such as weightlifting were associated with greater incidence of degenerative discs than runners. ${ }^{15}$

\section{INTERVENTION STRATEGIES}

The most common cause of LBP in athletes are related to sprains or strains; however, chronic and recurrent symptoms are frequently associated with degenerative lumbar disc disease and disc herniation. Strategies to alleviate LBP associated with degenerative disc disease or herniation depend upon whether the patient has acute or chronic symptoms. The ultimate goal of treatment is decreased discomfort and restoration of function, with focus on eventual return to competition without injury reoccurrence. This section will highlight key medical treatments, with logical progression from the more conservative acute interventions (non-operative) to those of an invasive origin (surgical).

\section{Acute Phase}

During the initial stage of back pain resulting from degenerative disc conditions, rest from physical/sporting activity is a necessity; however, general rest should only be advised for the first few days, as prolonged bed rest has been shown to be detrimental to the patient. ${ }^{22}$ In addition to rest, pharmacotherapy treatments such as non-steroidal anti-inflammatory drugs (NSAIDs) may be administered depending upon the severity of pain. In addition to decreasing inflammation, analgesia is a major goal of NSAIDs use in the acute phase of injury. Lumbar supports have been proposed during the acute phase; evidence in the literature suggests that wearing lumbar supports improves one's pain score. ${ }^{23}$ However, wearing lumbar supports in the long term it is not often advised as it is known to promote disuse atrophy of the trunk muscles. Lumbar supports include corsets and belts that are commonly worn during the acute stage. Similar to degenerative disc disease, the mainstay of treatment for a herniated disc include physical therapy rehabilitation, relative rest, and NSAIDs.

In addition, the administration of a corticosteroid preparation to reduce inflammation may be used by injecting locally at the site of inflammation through epidural steroid injection. The use of epidural steroid injections in professional athletes is supported by a recent retrospective study. ${ }^{24}$ Oral preparations may also be prescribed, although there is little literature to support this. During this initial phase, there is a need to reduce pain associated with the disc herniation by decreasing the extent of nerve root irritation. For both discogenic deterioration and herniation, emphasis should be made on maintaining full range of motion of the lumbar spine with exception of excessive flexion and rotation, whilst decreasing associative pain and inflammation.

\section{Rehabilitative Exercise}

Although the rehabilitation of athletes follows a similar pattern to that of non-athletes, the exercises must however prepare athletic populations for mechanical spinal loads often seen in many sports. Essentially, rehabilitative exercise is part of nonoperative measures to prevent and alleviate symptoms in spinal pathologies, although post-surgery would also require physical therapy. It is important to highlight that the correct prescription of exercise for spinal pathologies requires knowledge of the trunk and its supporting muscles.

These include paraspinal (multifidus, quadratum lumborum, erector spinae) and abdominal (transversus abdominis, and external and internal obliques) muscles. External loads to the spine are resisted by ligamentous structures; however, additional support is required form the trunk musculature. Watkins and Watkins proposed a trunk stabilisation exercise programme using a combination of special isometric strengthening exercises to develop important trunk muscles. ${ }^{25}$ Exercise is started once the athlete can tolerate physical activity, and the primary aim is to bring the spine back to a position of balance and power in injured athletes. The emphasis is upon the combination of muscle balance, coordination, and strength. This trunk stabilization prescription allows the athlete to move in incremental steps through increasingly advanced exercises. A more specific and progressive rehabilitative exercise programme in athletes with discogenic lumbar pain was described by Cooke and Lutz; this is illustrated in figure 1.26

(C) The Internet Journal of Allied Health Sciences and Practice, 2014 


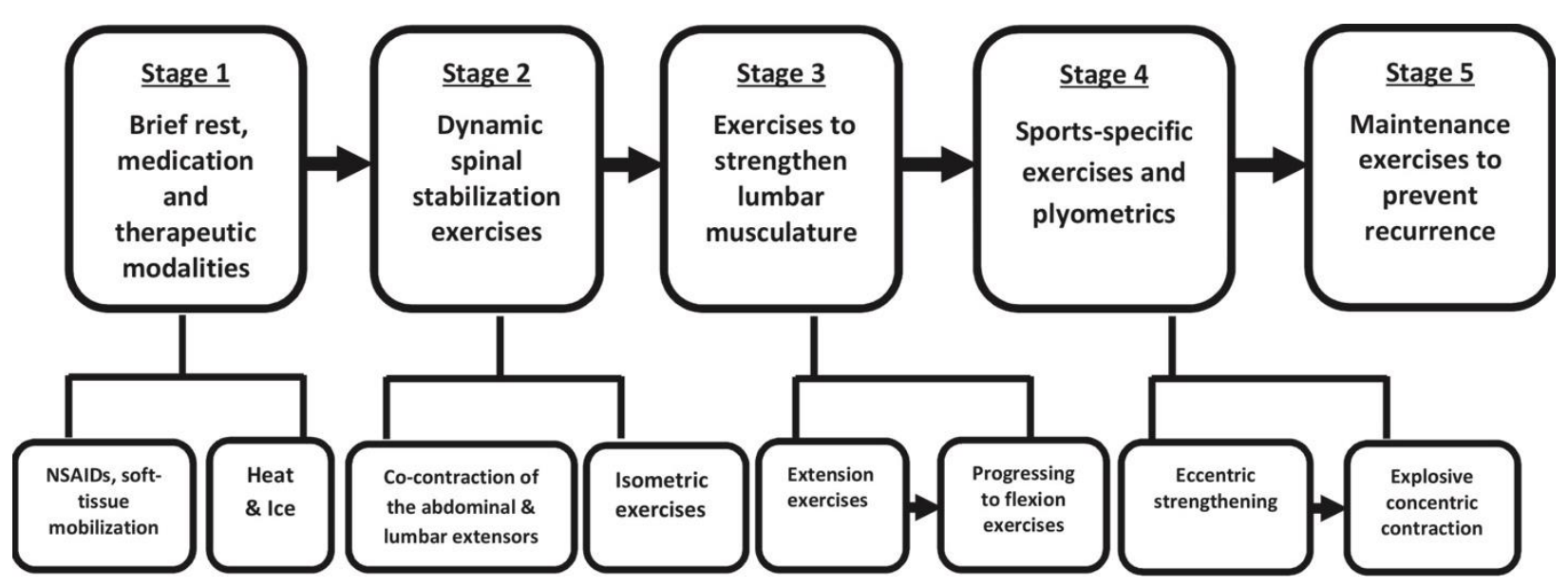

Figure 1. Guidelines for the prescription of an exercise programme in those with discogenic back pain.

The rehabilitation process may consider surface electromyographical (EMG) assessment. This should be conducted at regular intervals throughout the rehabilitation of the athlete, which will allow the clinician useful information in terms of the effectiveness of the prescribed exercise on muscle functions. Decreased stability of the lumbar spine has been identified as a potential risk factor for the development of lower back pathologies.

Appropriate spinal stability relies upon the combination of trunk muscle forces and appropriately timed muscle activity. ${ }^{27}$ Electromyographical assessment of those with LBP has been associated with reductions in the function of the abdominals and paraspinal muscles ${ }^{28}$ These neuromuscular alterations have been attributed to reduced spinal stability by deficits in muscular strength and endurance..$^{29} \mathrm{EMG}$ may therefore provide valuable information in relation to the function of the muscles that provide lumbar spinal stabilization during sports specific manoeuvres and thus could be useful in the determination of return to sports after spinal injuries.

\section{CHRONIC OR PERSISTENT SYMPTOMS}

Chronic or persistent conditions may require more invasive interventions and should only be considered if the athlete reports persistent, intolerable pain despite the application of aggressive non-operative therapies.

Discectomy may be considered as a surgical treatment in athletes. Essentially, discectomy is the surgical procedure which removes discogenic material that may be applying undue force upon a nerve root or the spinal cord. A recent review found that conservative treatment, or microdiscectomy, in athletes with lumbar disc herniation seemed to be satisfactory in terms of their ability to return the injured athletes to their original levels of sports activities. ${ }^{30} \mathrm{~A}$ study by Anakwenze et al investigating open discectomy in National Basketball Association (NBA) players demonstrated that $75 \%$ of patients returned to play again compared with $88 \%$ in control subjects who did not undergo the surgery. ${ }^{31}$ Furthermore, for those players who returned, overall athletic performance was slightly improved or no worse than control subjects. More recently, Hsu et al conducted a retrospective study in 226 athletes who underwent lumbar discectomy surgery from 4 major sports. ${ }^{32}$ Of those who had surgery, $81 \%$ returned to sport, in an average length of career after surgery being 3.3 years.

Spinal fusion is another surgical treatment available to athletes; however, this should only be considered if all other treatments fail to rectify the athlete's pain. This method is not very popular to use in athletes, and return to collision sports is generally not recommended. To our knowledge, there has been only one report on the results of lumbar disc fusion in athletes. A recent retrospective study by Fabricant et al investigated return to play in forty-two athletically active adolescents who underwent posterior spinal fusion for adolescent idiopathic scoliosis. It was found that $59.5 \%$ had returned to sports at an equal or higher level of physical activity. ${ }^{33}$

Lumbar disc replacement is the final operative procedure to be described within this report. Total lumbar disc replacement is performed predominately in young, active patients as there are previously defined contraindications with regards to age and disc replacement. ${ }^{34}$ To date, only one study was found that investigated the success of lumbar disc replacement in an athletic population. Siepe et al conducted a prospective study in 39 patients that participated significantly in sporting activities; 
importantly, the inclusion criteria also included those who participated in a variety of extreme sports with exposure to either repetitive forces or high-impact external loads. ${ }^{35}$ Thirty-seven subjects successfully returned to sporting activity, with 33 showing improvements in performance. Minor subsidence was observed in 13 patients within the first 3 months, although this trend was not continued after this period. However, the implants resilience to impact during physical activities has not been established yet. There are concerns regarding forces to the implant in particular sports that require the athlete to perform repetitive axial/rotational stress or sudden exposure to high external forces. Therefore, longer follow-up studies need to be considered in order to evaluate the durability and resilience of implants.

\section{RECOMMENDATIONS FOR RETURN TO PRACTICE \& COMPETITION}

There are numerous considerations to be made when deciding when safe return to practice or competition following an injury for an athlete is possible. The decision processes should be based on the injury status, ability to successfully perform sports-specific movements, and the psychological state of the athlete. These will be influenced by the effectiveness of the treatment received by the athlete and are applicable to both conservative and surgical procedures to rectify lumbar disc herniation or disc degeneration. Furthermore, clinicians should make careful clinical observations before allowing athletes with discogenic associated back pain to return to sports. The athlete should present with full range of motion in all anatomical axes without pain, maintain a neutral spine position during sports- specific movements, and display a return of muscular control, endurance and strength ${ }^{26}$ In addition to these guidelines, restoration of flexibility should be achieved in those athletes with herniated lumbar discs.

Following surgical interventions, there exist number of factors which dictate return to competition or training for the athlete, including type of surgery performed, age, gender, quality of surgical procedure, rehabilitative exercise correction, and motivational levels of the performer. ${ }^{36}$ Elite athletes are more likely to return to play than their non-elite counterparts.

A recent review by Iwamoto et al into athletic populations with herniated discs found that return to competition was 4.7 months for conservative intervention and 5.2 to 5.8 for microdiscectomy. ${ }^{30}$ This indicates that conservative treatment or microdiscectomy in athletes with lumbar disc herniation appeared to be satisfactory in terms of their ability to return to play. Following lumbar microdiscectomy in athletes, Kahanovitz proposes the return to non-contact sports depends upon the intensity, with more demanding tasks requiring 6 weeks, whereas less demanding sports only 4 weeks. ${ }^{37}$ Contact sports require 8 to 9 weeks before returning to competition. Eck and Riley recommend 6 to 8 weeks post- microdiscectomy before return to non-contact sports and 4 to 6 months before return to contact sports. ${ }^{38}$ Watkins et al found that 53 of 60 Olympic and professional athletes returned to previous level of competition, in an average time of 5.2 months after microdiscectomy. ${ }^{39}$

Following lumbar fusion, the time scale required for a successful return to sports lacks evidence. Only one report documents the results of lumbar fusion in athletes. ${ }^{33}$ It has been suggested that return to non-contact sports could be achieved within 6 to 12 months post-surgery, although this recommendation has no evidence to support it to date. 40 Essentially, the return to sports may be considered with convincing evidence of complete fusion, a restoration of both range of motion and strength, and no indication of pain.

Lumbar disk-replacement surgery is alternative to fusion in athletes and has become a more recent treatment for non-athletic patients. Siepe et al reported their findings in 39 athletes following lumber disc replacement. ${ }^{35}$ The return to non-contact sports was recommended after 3 month rehabilitation period, whereas more demanding and contact sports may be resumed between 4 to 6 months post-surgery. In addition, the author's state that solid osteointegration of implants allow for further load increases.

\section{CONCLUSION}

Depending on the severity of symptoms, it is apparent that athletes with lumbar disc herniation and signs of disc degeneration will benefit from more conservative treatments. What is crucial for the athlete is the correct conservative treatment at the appropriate times. Rehabilitative exercise plays a vital role in determining the time required in returning to either physical training or competition, and thus the correct exercise prescription is extremely important. Equally as important is the frequent monitoring of the success of the rehabilitation programme, not only through measurements of pain and range of motion, but also with EMG analysis of the involved muscles.

The clinician must also consider situations in which athletes still present pain symptoms even after vigorous conservative interventions. These patients may require surgical procedures in order to achieve the goal of becoming pain free. Although surgery can frequently correct the athletic injury, there remains the issues of whether surgical correction is sufficient to allow the athlete to return to the rigors of the sport, and if so, whether it will take weeks, months,or years to make that return. Spinal fusion may present serious drawbacks for athletic populations, especially for those who participate in contact orientated sports.

(C) The Internet Journal of Allied Health Sciences and Practice, 2014 
Thus, this treatment should be considered when other treatments have failed to correct the spinal complaints of the athlete. Lumbar disc replacement should also only be considered once the athlete clearly fails to respond to conservative measures, as there are concerns for the durability of the implant, especially during excessive spinal loading conditions. Discectomy provides a more suitable surgical intervention, with a significant number of athletes returning to sport at pre-injury performance levels. In general, specific guidelines for return to practice or competition remain poorly defined, with various estimates of the time needed. The clinician must decide carefully when it will be safe for the athlete to return to his/her sport(s). This would be once appropriate rehabilitation has been delivered and after the patient can successfully demonstrate pain-free range of motion during movements that mimic those of the their sport.

Therefore, the return to sports for physically active populations should be based on individual cases without encouraging a premature return, which could result in further injuries.

\section{REFERENCES}

1. Leone A, Guglielmi G, Cassar-Pullicino VN, Bonomo L. Lumbar intervertebral instability: a review. Radiology. Oct 2007;245(1):62-77. [PMID 17885181]

2. Urban JP, Roberts S. Degeneration of the intervertebral disc. Arthritis Research \& Therapy. 2003;5(3):120-30. [PMID 12723977]

3. Modic MT, Ross JS. Lumbar degenerative disk disease. Radiology. Oct 2007;245(1):43-61. [PMID 17885180]

4. Boos N, Weissbach S, Rohrbach H, Weiler C, Spratt KF, Nerlich AG. Classification of age-related changes in lumbar intervertebral discs: 2002 Volvo Award in basic science. Spine. Dec 1 2002;27(23):2631-44. [PMID 12461389]

5. Miller JA, Schmatz C, Schultz AB. Lumbar disc degeneration: correlation with age, sex, and spine level in 600 autopsy specimens. Spine. Feb 1988;13(2):173-8. [PMID 3406837]

6. Ahmed AM, Duncan NA, Burke DL. The effect of facet geometry on the axial torque-rotation response of lumbar motion segments. Spine. May 1990;15(5):391-401. [PMID 2363067]

7. Smeal WL, Tyburski M, Alleva J, Prather H, Hunt D. Conservative management of low back pain, part I. Discogenic/radicular pain. Disease-A-Month:DM. Dec 2004;50(12):636-69. [PMID 15767994]

8. Granhed $\mathrm{H}$, Jonson R, Hansson T. The loads on the lumbar spine during extreme weight lifting. Spine. Mar 1987;12(2):146-9. [PMID 3589805]

9. Moorman CT 3rd, Johnson DC, Pavlov H, et al. Hyperconcavity of the lumbar vertebral endplates in the elite football lineman. The American Journal of Sports Medicine. Sep 2004;32(6):1434-9. [PMID 15310568]

10. Dimitriadis AT, Papagelopoulos PJ, Smith FW, et al. Intervertebral disc changes after $1 \mathrm{~h}$ of running: a study on athletes. The Journal of International Medical Research. 2011;39(2):569-79. [PMID 21672362]

11. Adams MA, Freeman BJ, Morrison HP, Nelson IW, Dolan P. Mechanical initiation of intervertebral disc degeneration. Spine. Jul 1 2000;25(13):1625-36. [PMID 10870137]

12. Shankar H, Scarlett JA, Abram SE. Anatomy and pathophysiology of intervertebral disc disease. Techniques in Regional Anesthesia and Pain Management. 2009;13(2):67-75.

13. Moore RJ, Vernon-Roberts B, Fraser RD, Osti OL, Schembri M. The origin and fate of rniated lumbar intervertebral disc tissue. Spine. Sep 15 1996;21(18):2149-55. [PMID 8893441]

14. Adams MA, Hutton WC. Prolapsed intervertebral disc. A hyperflexion injury 1981 Volvo Award in Basic Science. Spine. May-Jun 1982;7(3):184-91. [PMID 7112236]

15. Videman T, Sarna S, Battie MC, et al. The long-term effects of physical loading and exercise lifestyles on back-related symptoms, disability, and spinal pathology among men. Spine. Mar 15 1995;20(6):699-709. [PMID 7604346]

16. Bono CM. Low-back pain in athletes. The Journal of Bone and Joint Surgery. American volume. Feb 2004;86-A(2):38296. [PMID 14960688]

17. Sward L, Hellstrom M, Jacobsson B, Nyman R, Peterson L. Disc degeneration and associated abnormalities of the spine in elite gymnasts. A magnetic resonance imaging study. Spine. Apr 1991;16(4):437-43. [PMID 1828629]

18. Bennett DL, Nassar L, DeLano MC. Lumbar spine MRI in the elite-level female gymnast with low back pain. Skeletal radiology. Jul 2006;35(7):503-9. [PMID 16520993]

19. Ong A, Anderson J, Roche J. A pilot study of the prevalence of lumbar disc degeneration in elite athletes with lower back pain at the Sydney 2000 Olympic Games. British Journal of Sports Medicine. Jun 2003;37(3):263-6. [PMID 12782554]

20. Alyas F, Turner M, Connell D. MRI findings in the lumbar spines of asymptomatic, adolescent, elite tennis players. British Journal of Sports Medicine. Nov 2007;41(11):836-41; [PMID 17640926] discussion 841.

21. Capel A, Medina FS, Medina D, Gomez S. Magnetic resonance study of lumbar disks in female dancers. The American Journal of Sports Medicine. Jun 2009;37(6):1208-13. [PMID19417120]

(c) The Internet Journal of Allied Health Sciences and Practice, 2014 
22. Saal JA. Natural history and nonoperative treatment of lumbar disc herniation. Spine. Dec 15 1996;21(24 Suppl):2S9S. [PMID 9112320]

23. Sato N, Sekiguchi M, Kikuchi S, Shishido H, Sato K, Konno S. Effects of long-term corset wearing on chronic low back pain. Fukushima Journal of Medical Science. 2012;58(1):60-5. [PMID 22790893]

24. Krych AJ, Richman D, Drakos M, et al. Epidural steroid injection for lumbar disc herniation in NFL athletes. Medicine and Science in Sports and Exercise. Feb 2012;44(2):193-8. [PMID 21760554]

25. Watkins RG, Watkins IRG. Lumbar Spondylolysis and Spondylolisthesis in Athletes. Seminars in Spine Surgery. 2010;22(4):210-7.

26. Cooke PM, Lutz GE. Internal disc disruption and axial back pain in the athlete. Physical Medicine and Rehabilitation Clinics of North America. Nov 2000;11(4):837-65. [PMID 11092021]

27. Cholewicki J, Panjabi MM, Khachatryan A. Stabilizing function of trunk flexor-extensor muscles around a neutral spine posture. Spine. Oct 1 1997;22(19):2207-12. [PMID 9346140]

28. Hodges PW, Richardson CA. Altered trunk muscle recruitment in people with low back pain with upper limb movement at different speeds. Arch Phys Med Rehabil. Sep 1999;80(9):1005-12. [PMID 10489000]

29. Hodges PW. The role of the motor system in spinal pain: implications for rehabilitation of the athlete following lower back pain. Journal of Ccience and Medicine in Sport / Sports Medicine Australia. Sep 2000;3(3):243-53. [PMID 11101263]

30. Imamoto J, Sato $\mathrm{Y}$, Takeda $\mathrm{T}$, Matsumoto $\mathrm{H}$. The return to sports activity after conservative or surgical treatment in athletes with lumbar disc herniation. American Journal of Physical Medicine \& Rehabilitation / Association of Academic Physiatrists. Dec 2010;89(12):1030-5.

31. Anakwenze OA, Namdari S, Auerbach JD, et al. Athletic performance outcomes following lumbar discectomy in professional basketball players. Spine. Apr 1 2010;35(7):825-8. [PMID 20195192]

32. Hsu WK, McCarthy KJ, Savage JW, et al. The Professional Athlete Spine Initiative: outcomes after lumbar disc herniation in 342 elite professional athletes. The Spine Journal: Official Journal of the North American Spine Society. Mar 2011;11(3):180-6. [PMID 21269889]

33. Fabricant PD, Admoni S, Green DW, Ipp LS, Widmann RF. Return to athletic activity after posterior spinal fusion for adolescent idiopathic scoliosis: analysis of independent predictors. Journal of Pediatric Orthopedics. Apr-May 2012;32(3):259-65. [PMID 22411331]

34. Huang RC, Lim MR, Girardi FP, Cammisa FP Jr. The prevalence of contraindications to total disc replacement in a cohort of lumbar surgical patients. Spine. Nov 2004;29(22):2538-41. [PMID 15543070]

35. Siepe CJ, Wiechert K, Khattab MF, Korge A, Mayer HM. Total lumbar disc replacement in athletes: clinical results, return to sport and athletic performance. European spine journal : official publication of the European Spine Society, the European Spinal Deformity Society, and the European Section of the Cervical Spine Research Society. Jul 2007;16(7):1001-13.

36. Iwamoto J, Sato Y, Takeda T, Matsumoto H. Return to play after conservative treatment in athletes with symptomatic lumbar disc herniation: a practice-based observational study Open Access Journal of Sports Medicine. 2011;2:25-31.

37. Kahanovitz N. Surgical disc excision. Clinics in Sports Medicine. Jul 1993;12(3):579-85. [PMID 8364994]

38. Eck JC, Riley LH, 3rd. Return to play after lumbar spine conditions and surgeries. Clinics in Sports Medicine. Jul 2004;23(3):367-79, viii. [PMID 15262376]

39. Watkins RG, Williams LA, Watkins RG, 3rd. Microscopic lumbar discectomy results for 60 cases in professional and Olympic athletes. The Spine Journal:Official Journal of the North American Spine Society. Mar-Apr 2003;3(2):100-5. [PMID 14589222]

40. Wright A, Ferree B, Tromanhauser S. Spinal fusion in the athlete. Clinics in Sports Medicine. Jul 1993;12(3):599-602. [PMID 8364996] 